\title{
ZigZAG INEQUALITIES: A NEW CLASS OF FACET-INDUCING inequalities for Arc Routing Problems
}

\author{
Angel Corberán ${ }^{1 *}$ Isaac Plana ${ }^{1}$ and José M. Sanchis ${ }^{2}$ \\ ${ }^{1}$ Dept. d'Estadística i Investigació Operativa, Universitat de València, Spain \\ ${ }^{2}$ Dept. de Matemática Aplicada, Universidad Politécnica de Valencia, Spain
}

July 19, 2005

\begin{abstract}
In this paper we introduce a new class of facet-inducing inequalities for the Windy Rural Postman Problem and the Windy General Routing Problem. These inequalities are called Zigzag inequalities because they cut off fractional solutions containing a zigzag associated with variables with 0.5 value. Two different types of inequalities, the Odd Zigzag and the Even Zigzag inequalities, are presented. Finally, their application to other known Arc Routing Problems is discussed.
\end{abstract}

Key Words: Polyhedral Combinatorics, Facets, Arc Routing, Windy Rural Postman Problem, Windy General Routing Problem, Mixed Rural Postman Problem.

\section{Introduction}

Arc Routing Problems consist of finding a shortest tour (closed walk) on the links of a given graph $G$ satisfying certain conditions. Graph $G$ can be undirected (all the links are edges that can be traversed, at the same cost, in both directions), directed (all the links are arcs that must be traversed in a given direction) or mixed (having edges and arcs simultaneously). Obviously, routing problems defined on mixed graphs generalize the corresponding problems defined on undirected and directed graphs. Finally, we have the 'Windy' Arc Routing Problems, which are defined on an undirected graph where the cost of traversing an edge in a given direction can be different to the cost of traversing it in the opposite direction. These problems generalize those defined on mixed graphs because an $\operatorname{arc}(i, j)$ with cost $c$ can be modelled as an edge with costs $c_{i j}=c$ and $c_{j i}=\infty$.

In this paper we deal with the Windy General Routing Problem, WGRP. This problem can be defined as follows. Let $G=(V, E)$ be an undirected and connected graph with two non-negative costs $c_{i j}$ and $c_{j i}$ associated with each edge $\{i, j\} \in E$ corresponding to the cost of traversing it from $i$ to $j$ and from $j$ to $i$, respectively. Given a subset $E_{R} \subseteq E$ of 'required' edges and a given subset $V_{R} \subseteq V$ of 'required' vertices, the objective is to find the tour on $G$ traversing each 'required' edge and each 'required' vertex at least once, at minimum cost.

This is a difficult combinatorial optimization problem that generalizes several well known $N P$-hard Node and Arc Routing Problems. Note that if $E_{R}=\emptyset$ and $V_{R}=V$, we have as

\footnotetext{
*corresponding author: angel.corberan@uv.es
} 
special cases the Graphical Traveling Salesman Problem (Cornuèjols, Fonlupt \& Naddef, 1985, Naddef \& Rinaldi, 1991) when $c_{i j}=c_{j i} \quad \forall\{i, j\} \in E$, and the Graphical Asymmetric Traveling Salesman Problem (Chopra \& Rinaldi, 1996) otherwise. On the other hand, if $V_{R}=\emptyset$, the WGRP reduces to the Windy Rural Postman Problem and if in addition $E_{R}=E$, to the Windy Postman Problem.

The Windy Postman Problem, WPP, was first introduced by Minieka (1979). This is an NPhard problem (Guan, 1984) that was also studied by Win (1987) and by Grötschel \& Win (1988, 1992). Notice that it contains the undirected, directed and mixed versions of the well-known Chinese Postman Problem (CPP, DCPP and MCPP, respectively) as particular cases. The Windy Rural Postman Problem, WRPP, has been studied in Benavent et al. (2003, 2005). In these papers, several heuristic algorithms are presented, as well as a formulation of the problem and several families of valid linear inequalities. A polyhedral study of the WGRP has been done by Corberán, Plana and Sanchis (2005). The WRPP formulation in Benavent et al. (2003) and the WGRP formulation in Corberán et al. (2005) are exactly the same. Hence, from the point of view of polyhedral combinatorics, both problems can be considered equivalent and therefore the WGRP can be seen as an Arc Routing Problem.

Since some Arc Routing Problems on mixed graphs are particular cases of the Windy General Routing Problem, they can be handled with a similar formulation. This formulation uses two variables associated with each edge, representing the number of times the edge is traversed in each direction and was used, for example, by Christofides et al. (1984) and Ralphs (1993) for the Mixed Chinese Postman Problem. Furthermore, these problems can also be formulated using only one variable for each edge, representing the number of times a given edge is traversed (in any direction). This approach was first proposed by Nobert \& Picard (1996) for the MCPP and more recently by Corberán, Romero \& Sanchis (2003) and by Corberán, Mejía \& Sanchis (2005) for the Mixed Rural Postman Problem (MRPP) and the Mixed General Routing Problem (MGRP).

When we use a cutting-plane algorithm to solve the WGRP or the MGRP (with either of both formulations), fractional solutions satisfying all the known facet-inducing inequalities are found for some instances. These fractional solutions have the 'typical' common feature of containing closed walks associated with variables with 0.5 'extra' value, which often look like a zigzag. We have found a new class of facet-inducing inequalities which cut off this kind of solution. The purpose of this paper is to present this new family of inequalities for the Windy and Mixed General Routing Problems, which also applies to other Arc Routing Problems. These inequalities generalize the 3-wheel inequalities proposed by Win (1987) for the WPP.

In section 2 the WGRP is defined and several known results are summarized. Section 3 describes two different versions of Zigzag inequalities for the WGRP, the odd case and the even case. Section 4 is devoted to the application of these inequalities to other known Arc Routing Problems. In section 5 some computational experiments are discussed and section 6 presents the conclusions.

\section{Problem definition and known results}

As mentioned above, the Windy General Routing Problem consists of finding a minimum cost closed walk traversing all the required edges, $E_{R} \subseteq E$, and visiting all the required vertices, $V_{R} \subseteq V$, of an undirected graph $G=(V, E)$ (WGRP tour). We can suppose w.l.o.g. that $V_{R}=V$, because it is easy to transform an instance which does not satisfy this condition into an instance that does (see, for example, Christofides et al., 1981, or Eiselt, Gendreau \& Laporte, 
1995). The graph $G_{R}=\left(V, E_{R}\right)$ is, in general, non-connected. Let us denote by $p$ the number of its connected components and by $V_{1}, V_{2}, \ldots, V_{p}$, with $V_{1} \cup \ldots \cup V_{p}=V$, their corresponding vertex sets. Given a node subset, $S \subseteq V$, let $\delta(S)$ denote the edge set with an end-point in $S$ and the other in $V \backslash S$ and let $E(S)$ be the set of edges with both end-points in $S$. Given two node subsets $S_{1}, S_{2} \subseteq V,\left(S_{1}, S_{2}\right)$ will represent the set of edges with one end-point in $S_{1}$ and the other in $S_{2}$, while $\delta_{R}(S), E_{R}(S),\left(S_{1}, S_{2}\right)_{R}$ will denote the previous sets, referring only to the required edges. Finally, a vertex is called $R$-even ( $R$-odd) if it is incident with an even (odd) number of required edges.

Let $x_{i j}$ be the number of times edge $\{i, j\}$ is traversed from $i$ to $j$ in a WGPP tour. Given $F \subseteq E$, we denote by $x(F)=\sum_{\{i, j\} \in F}\left(x_{i j}+x_{j i}\right)$. The formulation given by Benavent et al. (2003) for the WGRP is:

$$
\begin{array}{cll}
\text { Minimize } & \sum_{\{i, j\} \in E}\left(c_{i j} x_{i j}+c_{j i} x_{j i}\right) \\
\text { s.t.: } & & \\
x_{i j}+x_{j i} \geq 1, & \forall\{i, j\} \in E_{R} & \\
\sum_{\{i, j\} \in \delta(i)}\left(x_{i j}-x_{j i}\right)=0, & \forall i \in V & \\
\sum_{i \in S, j \in V \backslash S} x_{i j} \geq 1, & \forall S=\cup_{k \in Q} V_{k}, \quad Q \subset\{1, \ldots, p\} \\
x_{i j}, x_{j i} \geq 0, & \forall\{i, j\} \in E & \\
x_{i j}, x_{j i} \text { integer, } & \forall\{i, j\} \in E
\end{array}
$$

where conditions (2) and (3) force the (directed) graph represented by the tour to be, respectively, symmetric and connected, and conditions (1) imply that each required edge will be traversed. The above system includes an equation associated with each vertex. The $|V|$ equations (2) will be referred to as the system equations and any $|V|-1$ of them are linearly independent.

Let $\operatorname{WGRP}(G)$ be the convex hull of all the tours $x \in \mathbb{Z}^{2|E|}$ satisfying (1) to (4). In Corberán, Plana \& Sanchis (2005) it is shown that $\operatorname{WGRP}(G)$ is an unbounded polyhedron with dimension $2|E|-|V|+1$, and that the following inequalities are, under mild conditions, facet-inducing for $\operatorname{WGRP}(G)$ :

- Trivial inequalities (4)

- Traversing inequalities (1)

- Connectivity inequalities (3)

- The $R$-odd cut inequalities:

$$
x(\delta(S)) \geq\left|\delta_{R}(S)\right|+1, \forall \delta(S) \quad R \text {-odd cutset of } G
$$

- The (standard) $\mathrm{KC}$ and the $\mathrm{KC}_{02}$ inequalities (see Corberán, Romero \& Sanchis, 2003).

- The (standard) Path-Bridge (PB) and the Path-Bridge $02\left(\mathrm{~PB}_{02}\right)$ inequalities (see Letchford, 1997, and Corberán, Romero \& Sanchis, 2003).

- The (standard) Honeycomb and the Honeycomb 02 inequalities (see Corberán, Mejía \& Sanchis, 2005).

In the above-mentioned paper it is also shown that all these inequalities except (4) and (1) are configuration inequalities (Naddef \& Rinaldi, 1991), i.e. each inequality is defined by a partition of $V, \mathcal{B}=\left\{B_{1}, B_{2}, \ldots, B_{r}\right\}$, and by some costs associated with ordered pairs of node 
sets $B_{i}$. The variables associated with the edges in $E\left(B_{i}\right)$ have coefficient zero in the inequality, and the variables $x_{u v}, x_{v u}$ associated with the edges $\{u, v\} \in\left(B_{i}, B_{j}\right)$ have coefficients equal to the costs $c\left(B_{i}, B_{j}\right)$ and $c\left(B_{j}, B_{i}\right)$, respectively. However, it is worth noticing that, unlike other Arc Routing Polyhedra, $\operatorname{WGRP}(G)$ has facet-inducing inequalities that are not strictly configuration inequalities since they can have variables $x_{u v}, x_{s t}$, with $u, s \in B_{i}$ and $v, t \in B_{j}$, with different coefficients. These inequalities are called weak configuration inequalities. This is the case of the Zigzag inequalities presented in this paper. Associated with a (weak or standard) configuration inequality, we have a configuration graph, $G_{\mathcal{C}}$, having node set $\mathcal{B}$, a required edge $\left\{B_{i}, B_{j}\right\}$ for each required edge $\{u, v\}$ of $G$ with $u \in B_{i}, v \in B_{j}$ and a non-required edge $\left\{B_{i}, B_{j}\right\}$ for each pair $B_{i}, B_{j}$ such that $\left(B_{i}, B_{j}\right) \backslash E_{R} \neq \emptyset$. In other words, $G_{\mathcal{C}}$ is the graph resulting after shrinking node sets $B_{i}, i=1, \ldots, r$, into a single vertex each, and shrinking each set of non-required parallel edges into a single edge, but keeping all the required edges. Finally, in Corberán, Plana \& Sanchis (2005) a 'lifting' theorem providing conditions for a facet-inducing inequality for $\operatorname{WGRP}\left(G_{\mathcal{C}}\right)$ to be facet-inducing for $\operatorname{WGRP}(G)$, is also included.

\section{The Zigzag inequalities.}

Although the above inequalities describe the polyhedron $\operatorname{WGRP}(G)$ tightly, it is not unusual to find fractional solutions which satisfy all these inequalities and contain closed walks with edges whose associated variables have an extra value of 0.5, i.e. $x_{i j}+x_{j i}=1.5$ if $e_{i j} \in E_{R}$ and $x_{i j}+x_{j i}=0.5$ otherwise. Consider for example the WGRP instance in figure 1a, where the required edges are represented in bold lines and each number represents the cost of traversing the corresponding edge from the nearest node to the farthest node. Note that all the vertices are $R$-even. Consider also the vector $x^{*}$ represented in figure $1 \mathrm{~b}$, where arcs drawn in solid lines correspond to variables with value 1 while arcs in dotted lines correspond to variables with value 0.5 . This vector satisfies all the known inequalities and its cost is 54 , while the cost of an optimal WGRP tour is 62 . In fact, $x^{*}$ is an extreme point of the polyhedron defined by all the inequalities mentioned before. An inequality that is not satisfied by $x^{*}$ is

$F(x)=x_{13}+x_{31}+x_{13}^{\prime}+x_{31}^{\prime}+x_{24}+x_{42}+x_{24}^{\prime}+x_{42}^{\prime}+x_{14}+x_{41}+x_{23}+x_{32}+2 x_{21}+2 x_{43} \geq 6$

since $F\left(x^{*}\right)=5$. The coefficients of this inequality are shown in figure $1 \mathrm{c}$.

Consider now the WGRP instance in figure 2a, where all the vertices are $R$-odd. Again, it can be seen that the vector $x^{*}$ shown in figure $2 \mathrm{~b}$, where variables with value 1.5 are represented by a solid and a dotted arc simultaneously, is an extreme point of the above polyhedron, but $x^{*}$ is not a WGRP tour. An inequality that is not satisfied by $x^{*}$ is (see figure 2c):

$$
F(x)=x_{13}+x_{31}+x_{24}+x_{42}+x_{14}+x_{41}+x_{23}+x_{32}+2 x_{21}+2 x_{43} \geq 6
$$

since $F\left(x^{*}\right)=5$.

Both constraints belong to a new family of inequalities that will be called Zigzag inequalities. We show below that these inequalities are valid and facet-inducing for $\operatorname{WGRP}(G)$. Although the final form of these inequalities can be similar in some cases, the meaning of the inequalities and the conditions needed to be facet-inducing are quite different for the odd and even cases. Hence, we will distinguish between Even Zigzag inequalities (the four nodes are $R$-even) and Odd Zigzag inequalities (the four nodes are $R$-odd). 


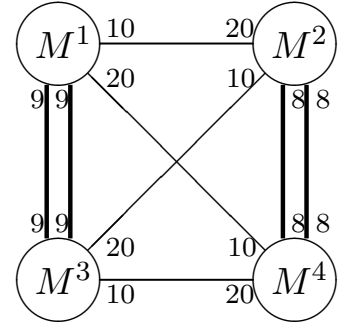

(a)

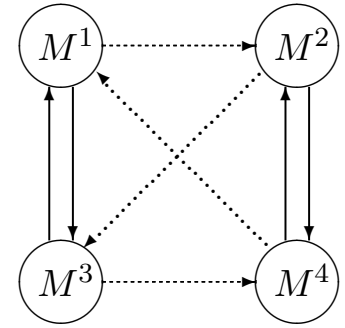

(b)

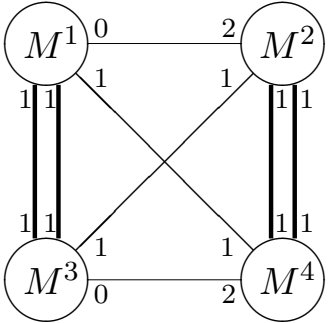

(c)

Figure 1: WGRP instance, fractional solution and Even Zigzag configuration.

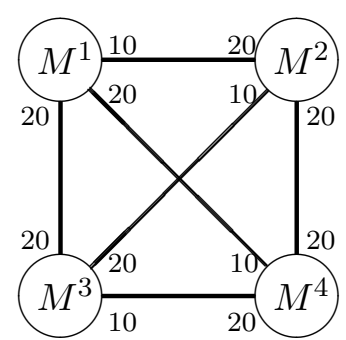

(a)

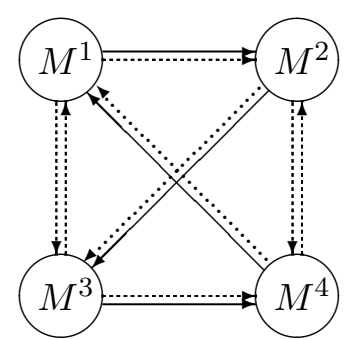

(b)

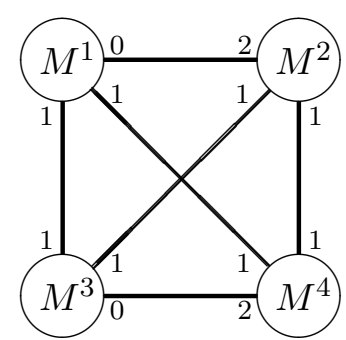

(c)

Figure 2: WGRP instance, fractional solution and Odd Zigzag configuration.

\subsection{Even Zigzag inequalities}

Consider a partition of the set of vertices $V$ into 4 parts, $M^{1}, M^{2}, M^{3}$ and $M^{4}$, where each $M^{i}$ contains an even number of R-odd vertices. Let $\alpha_{i j}$ denote the number of required edges in $\left(M^{i}, M^{j}\right)$ and suppose that the following condition is satisfied:

$$
\alpha_{12}=\alpha_{34}=\alpha_{14}=\alpha_{23}=0
$$

The configuration graph $G_{\mathcal{C}}$ associated with the Even Zigzag inequalities is defined by the partition of $V$ above and by the following coefficients (see figure 1c):

$$
c\left(M^{1}, M^{2}\right)=c\left(M^{3}, M^{4}\right)=0, \quad c\left(M^{2}, M^{1}\right)=c\left(M^{4}, M^{3}\right)=2, \quad c\left(M^{i}, M^{j}\right)=1, \text { otherwise. }
$$

Given a set of edges, $\left(S_{1}, S_{2}\right)$, we will denote by $x\left(S_{1}: S_{2}\right)=\sum_{\{i, j\}: i \in S_{1}, j \in S_{2}} x_{i j}$. We will call the following inequality Even Zigzag inequality

$$
F(x)=x\left(\delta\left(M^{1} \cup M^{2}\right)\right)+2 x\left(M^{2}: M^{1}\right)+2 x\left(M^{4}: M^{3}\right) \geq \alpha_{13}+\alpha_{24}+2
$$

Theorem 1 Even Zigzag inequalities (8) are valid for $W G R P(G)$.

Proof: Any WGRP tour $x$ must traverse all the required edges in one of its two possible directions. Given that the coefficient in the inequality of the two variables associated with these edges is equal to $1, x$ has at least an $F$-cost of $\alpha_{13}+\alpha_{24}$ on these variables. Furthermore, the tour $x$ must traverse the cutset $\delta\left(M^{1} \cup M^{3}\right)$ in both directions. This is done with $F$-cost greater than or equal to 2 . 
Theorem 2 Even Zigzag inequalities (8) are facet-inducing for $W G R P(G)$ if $G_{\mathcal{C}}$ is a complete graph and $\alpha_{13}, \alpha_{24} \geq 2$ (and even).

Proof: We will first prove that Zigzag inequalities are facet-inducing for $\operatorname{WGRP}\left(G_{\mathcal{C}}\right)$. The dimension of $\operatorname{WGRP}\left(G_{\mathcal{C}}\right)$ is twice the number of edges in $G_{\mathcal{C}}$ minus 3 . This is the number of linearly independent WGRP tours satisfying $F(x)=\alpha_{13}+\alpha_{24}+2$ we have to define. Each tour is a vector with two components $x_{i j}, x_{j i}$ associated with each edge $e=\{i, j\}$ in $G_{\mathcal{C}}$ expressing the number of times edge $e$ is traversed in each direction.

Given that $\alpha_{13}$ and $\alpha_{24}$ are even, it is possible to direct all the required edges in $G_{\mathcal{C}}$ in such a way that they induce a (directed) symmetric subgraph. Let $x^{d}$ denote the incidence vector of this subgraph, i.e. $x_{i j}^{d}=1$ if $e=\{i, j\}$ is a required edge that has been oriented from $i$ to $j$ and $x_{i j}^{d}=0$ otherwise. Note that $F\left(x^{d}\right)=\alpha_{13}+\alpha_{24}$.

We select the components corresponding to the 4 non-required edges $e_{12}, e_{23}, e_{34}$ and $e_{14}$ in the direction given by the zigzag, i.e. in the direction $M^{1}-M^{2}-M^{3}-M^{4}-M^{1}$. In what follows, we build a WGRP tour associated with each unselected component $x_{i j}$. Given an edge $e_{i j}, y\left(\overrightarrow{e_{i j}}\right)$ (respectively $y\left(\overleftarrow{e_{i j}}\right)$ ) denotes the vector with zero in all its components except for component $x_{i j}$ (respectively $x_{j i}$ ), which is equal to 1 . For each non-selected component $x_{i j}$ associated with an edge $e_{i j} \in \delta\left(M^{1} \cup M^{3}\right)$ we define the tour $x^{d}+y\left(\overrightarrow{e_{i j}}\right)+y\left(\overleftarrow{e_{i j}}\right)$. For each non-selected component of edges $e \in\left(M^{1}, M^{3}\right)$ we define the tour $x^{d}+y(\vec{e})+y\left(\overrightarrow{e_{34}}\right)+y\left(\overleftarrow{e_{14}}\right)$ or the tour $x^{d}+y(\overleftarrow{e})+y\left(\overrightarrow{e_{12}}\right)+y\left(\overrightarrow{e_{23}}\right)$. Similarly, for each non-selected component of edges $e \in\left(M^{2}, M^{4}\right)$ we define the tour $x^{d}+y(\vec{e})+y\left(\overrightarrow{e_{12}}\right)+y\left(\overleftarrow{e_{14}}\right)$ or the tour $x^{d}+y(\overleftarrow{e})+y\left(\overrightarrow{e_{23}}\right)+y\left(\overrightarrow{e_{34}}\right)$. We define a final tour using $x^{d}$ and the 4 selected components: $x^{d}+y\left(\overrightarrow{e_{12}}\right)+y\left(\overrightarrow{e_{23}}\right)+y\left(\overrightarrow{e_{34}}\right)+y\left(\overleftarrow{e_{14}}\right)$. All these tours satisfy $F(x)=\alpha_{13}+\alpha_{24}+2$. If we subtract $x^{d}$ from all the tours and arrange them in rows it is easy to see that the matrix has full rank. Hence, the Even zigzag inequalities are facet-inducing for WGRP $\left(G_{\mathcal{C}}\right)$. By applying the lifting theorem in Corberán, Plana \& Sanchis (2005), they are also facet-inducing for $\operatorname{WGRP}(G)$.

\subsection{Odd Zigzag inequalities}

In addition to the instance shown in figure 2, a more complex one will be used to introduce the Odd Zigzag inequalities. Consider the fractional solution depicted in figure 3a, corresponding to a WGRP instance in which all edges are required. Again, arcs drawn in solid lines correspond to variables with value 1 , arcs in dotted lines correspond to variables with value 0.5 and variables with value 1.5 are represented with a solid and a dotted arc simultaneously. This is also a typical solution where variables with an extra value of 0.5 form a zigzag. This kind of solution violates the Odd Zigzag inequalities that are presented in what follows.

Consider a partition of the set of vertices $V$ into 4 parts, $M^{1}, M^{2}, M^{3}$ and $M^{4}$, where each $M^{i}$ contains an odd number of $R$-odd vertices.

Let us call $\mathcal{H}=\left(M^{1}, M^{2}\right) \cup\left(M^{3}, M^{4}\right)$ (horizontal edges) and $\mathcal{D}=\left(M^{2}, M^{3}\right) \cup\left(M^{1}, M^{4}\right)$ (diagonal edges). Note that $\mathcal{H} \cup \mathcal{D}=\delta\left(M^{1} \cup M^{3}\right)$. Let us define a subset of required edges $\mathcal{F} \subset(\mathcal{H} \cup \mathcal{D})_{R}$ satisfying (see figure $3 \mathrm{~b}$, where edges in $\mathcal{F}$ are represented in bold lines)

$$
\left|\mathcal{H}_{R} \backslash \mathcal{F}\right|+\left|\mathcal{D}_{R} \cap \mathcal{F}\right|=\left|\mathcal{D}_{R} \backslash \mathcal{F}\right|+\left|\mathcal{H}_{R} \cap \mathcal{F}\right|
$$

The configuration graph $G_{\mathcal{C}}$ associated with the Odd Zigzag inequalities is defined by the partition of $V$ and the set $\mathcal{F}$ above, and by the following pair of coefficients associated with each edge $e_{i j}$ (see figure $3 \mathrm{~b}$ ): 


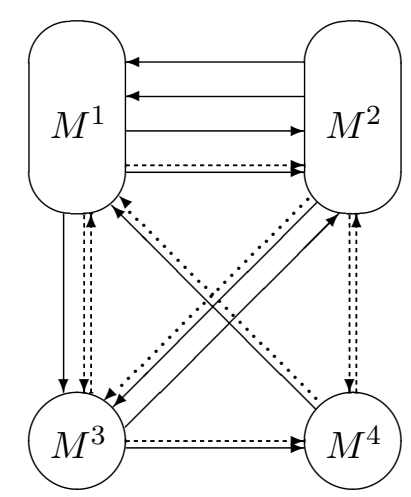

(a)

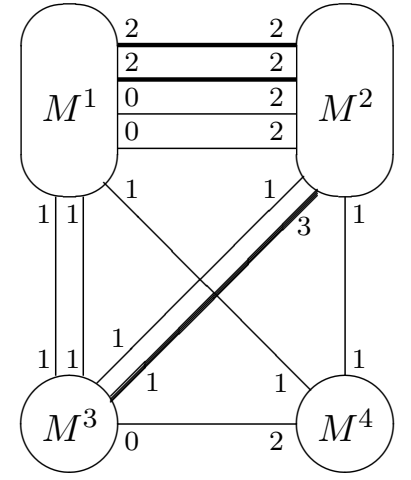

(b)

Figure 3: An Odd Zigzag configuration with set $\mathcal{F}$ in bold lines.

$$
\left(c_{i j}, c_{j i}\right)=\left\{\begin{array}{lll}
(0,2) & \forall e_{i j} \in \mathcal{H} \backslash \mathcal{F}, \quad i \in M^{1} \cup M^{3}, \quad j \in M^{2} \cup M^{4} \\
(2,2) & \forall e_{i j} \in \mathcal{H} \cap \mathcal{F} \\
(1,3) & \forall e_{i j} \in \mathcal{D} \cap \mathcal{F}, \quad i \in M^{1} \cup M^{3}, \quad j \in M^{2} \cup M^{4} \\
(1,1) & \text { otherwise }
\end{array}\right.
$$

The corresponding Odd Zigzag inequality is then

$$
x\left(\delta\left(M^{1} \cup M^{2}\right)\right)+2 x\left(M^{2}: M^{1}\right)+2 x\left(M^{4}: M^{3}\right)+2 x\left(F_{z z}\right) \geq \alpha_{13}+\alpha_{24}+\alpha_{14}+\alpha_{23}+2|\mathcal{H} \cap \mathcal{F}|+2
$$

where $x\left(F_{z z}\right)$ denotes the variables associated with the edges in $\mathcal{F}$ in the direction given by the zigzag.

Odd Zigzag inequalities (10) are in general weak configuration inequalities. However, when $\mathcal{F}=\emptyset$ they are standard configuration inequalities and their coefficients are equal to those of the Even Zigzag inequalities (see figure 2). Note that $\mathcal{F}=\emptyset$ is only possible when $\left|\mathcal{H}_{R}\right|=\left|\mathcal{D}_{R}\right|$.

Set $\mathcal{F}$ can be understood in the following way. The edges in $\delta_{R}\left(M^{1} \cup M^{3}\right)$ (an even number) can be oriented to obtain a (directed) symmetric subgraph. Given any such orientation, set $\mathcal{F}$ is defined by all the required edges that have been oriented in the opposite direction to the zigzag, i.e. in the direction $M^{4}-M^{3}-M^{2}-M^{1}-M^{4}$. In particular, set $\mathcal{F}$ in figure $3 \mathrm{~b}$ is defined from the orientation associated with the fractional solution shown in figure 3a. Other sets $\mathcal{F}$ can be defined to obtain valid inequalities but only the one shown in figure $3 \mathrm{~b}$ has an associated inequality violated by the fractional solution.

Theorem 3 Odd Zigzag inequalities (10) are valid for $W G R P(G)$.

Proof: Let $F(x) \geq c_{0}$ denote the inequality and let $x$ be a WGRP tour. Each WGRP tour must traverse every required edge in one of its two possible directions. Given that the coefficient in the inequality of the two variables associated with each edge in $\delta\left(M^{1} \cup M^{2}\right)$ is at least 1 , and those associated with each edge in $\mathcal{H} \cap \mathcal{F}$ is $2, x$ has at least an $F$-cost of $c_{0}-2$. If $x$ traverses any edge in $\mathcal{H} \backslash \mathcal{F}$ or in $\mathcal{D} \cap \mathcal{F}$ in the direction from $M^{2} \cup M^{4}$ to $M^{1} \cup M^{3}$, then it has an extra $F$-cost of 2 units and the inequality is satisfied. Therefore, we can suppose that $x$ traverses all the edges in $\mathcal{H}_{R} \backslash \mathcal{F}$ and in $\mathcal{D}_{R} \cap \mathcal{F}$ in the direction from $M^{1} \cup M^{3}$ to $M^{2} \cup M^{4}$ and, from (9), that $x$ traverses all the edges in $\mathcal{D}_{R} \backslash \mathcal{F}$ and in $\mathcal{H}_{R} \cap \mathcal{F}$ in the direction from $M^{2} \cup M^{4}$ to $M^{1} \cup M^{3}$. Since all the nodes are $R$-odd, the tour $x$ cannot traverse each required edge exactly once and 
an extra matching on the four nodes is needed. Notice that all the matchings have $F$-cost of at least two except the matching with $F$-cost zero defined by an edge not in $\mathcal{F}$ traversed in the direction from $M^{1}$ to $M^{2}$ and another from $M^{3}$ to $M^{4}$. But in this case the graph associated with $x$ is not symmetric.

Theorem 4 Odd Zigzag inequalities (10) are facet-inducing for $W G R P(G)$ if $G_{\mathcal{C}} \backslash \mathcal{F}$ is a complete graph, if there are two required edges $e_{13} \in\left(M^{1}, M^{3}\right)$ and $e_{24} \in\left(M^{2}, M^{4}\right)$ and if the remaining required edges in $G_{\mathcal{C}}$ can be oriented to induce a (directed) symmetric graph satisfying that:

(a) all the edges in $(\mathcal{H} \cup \mathcal{D})_{R} \backslash \mathcal{F}$ are oriented in the direction of the zigzag: $M^{1}-M^{2}-M^{3}-M^{4}-M^{1}$,

(b) all the edges in $\mathcal{F}$ are oriented in the opposite direction: $M^{4}-M^{3}-M^{2}-M^{1}-M^{4}$.

Proof: We will first prove that Odd Zigzag inequalities are facet-inducing for $\operatorname{WGRP}\left(G_{\mathcal{C}}\right)$. As for the even case, we need to find twice the number of edges in $G_{\mathcal{C}}$ minus 3 linearly independent WGRP tours satisfying $F(x)=c_{0}$. Again, each tour is a vector with two components $x_{i j}, x_{j i}$ associated with each edge $e=\{i, j\}$ in $G_{\mathcal{C}}$.

We first select the four components corresponding to the given required edges $e_{13}$ and $e_{24}$. Let $x^{d}$ denote the incidence vector of the symmetric subgraph induced by the orientation mentioned in the theorem. Note that the four selected components in $x^{d}$ are zero and that $F\left(x^{d}\right)=c_{0}-2$.

Since $G_{C} \backslash \mathcal{F}$ is a complete graph, it is possible to select another 4 components associated with 4 edges not in $\mathcal{F}, e_{12} \in\left(M^{1}, M^{2}\right), e_{23} \in\left(M^{2}, M^{3}\right), e_{34} \in\left(M^{3}, M^{4}\right)$ and $e_{14} \in\left(M^{1}, M^{4}\right)$, in the direction given by the zigzag. In what follows, we build a WGRP tour associated with each unselected component $x_{i j}$.

For each non-selected component associated with an edge $e \in\left(M^{1}, M^{2}\right) \backslash \mathcal{F}$ and depending on its direction, we define the tour $x^{1}=x^{d}+y(\vec{e})+y\left(\overleftarrow{e_{14}}\right)+y\left(\overrightarrow{e_{24}}\right)+y\left(\overrightarrow{e_{13}}\right)+y\left(\overleftarrow{e_{13}}\right)$ or the tour $x^{2}=x^{d}+y(\overleftarrow{e})+y\left(\overrightarrow{e_{34}}\right)+y\left(\overleftarrow{e_{24}}\right)+y\left(\overrightarrow{e_{13}}\right)$. For the non-selected components of each edge $e \in\left(M^{1}, M^{2}\right) \cap \mathcal{F}$, we define the tour $x^{3}=x^{d}+y(\vec{e})-y(\overleftarrow{e})+y\left(\overleftarrow{e_{14}}\right)+y\left(\overrightarrow{e_{24}}\right)+y\left(\overrightarrow{e_{23}}\right)+y\left(\overleftarrow{e_{13}}\right)$ or the tour $x^{4}=x^{d}+y(\overleftarrow{e})+y\left(\overrightarrow{e_{34}}\right)+y\left(\overleftarrow{e_{24}}\right)+y\left(\overrightarrow{e_{13}}\right)$. For the non-selected components of edges $e \in\left(M^{1}, M^{3}\right)$ we define the tour $x^{5}=x^{d}+y(\vec{e})+y\left(\overleftarrow{e_{13}}\right)+y\left(\overrightarrow{e_{24}}\right)+y\left(\overrightarrow{e_{12}}\right)+y\left(\overleftarrow{e_{14}}\right)$ or the tour $x^{6}=x^{d}+y(\overleftarrow{e})+y\left(\overrightarrow{e_{13}}\right)+y\left(\overrightarrow{e_{24}}\right)+y\left(\overrightarrow{e_{12}}\right)+y\left(\overleftarrow{e_{14}}\right)$. For edges $e \in\left(M^{2}, M^{3}\right) \backslash \mathcal{F}$, we define the tour $x^{7}=x^{d}+y(\vec{e})+y\left(\overrightarrow{e_{34}}\right)+y\left(\overleftarrow{e_{24}}\right)+y\left(\overrightarrow{e_{13}}\right)+y\left(\overleftarrow{e_{13}}\right)$ or the tour $x^{8}=x^{d}+y(\overleftarrow{e})+y\left(\overrightarrow{e_{24}}\right)+y\left(\overleftarrow{e_{14}}\right)+y\left(\overrightarrow{e_{13}}\right)$, and for edges $e \in\left(M^{2}, M^{3}\right) \cap \mathcal{F}$, the tour $x^{9}=x^{d}+y(\vec{e})-y(\overleftarrow{e})+y\left(\overrightarrow{e_{34}}\right)+y\left(\overleftarrow{e_{24}}\right)+y\left(\overrightarrow{e_{12}}\right)+y\left(\overleftarrow{e_{13}}\right)$ or the tour $x^{10}=x^{d}+y(\overleftarrow{e})+y\left(\overrightarrow{e_{24}}\right)+y\left(\overleftarrow{e_{14}}\right)+y\left(\overrightarrow{e_{13}}\right)$ are defined. For edges in $\left(M^{3}, M^{4}\right),\left(M^{2}, M^{4}\right)$ and $\left(M^{1}, M^{4}\right)$ we proceed as before. All these tours satisfy $F(x)=c_{0}$.

Finally, 5 more tours using $x^{d}$ and some of the 8 selected components are constructed. If we subtract $x^{d}$ from all the tours and arrange them in rows, the matrix in figure 4 is obtained. The first rows correspond to the tours associated with non-selected components in $\mathcal{F}$, while the last 5 rows correspond to the most-recently mentioned tours. Given that this matrix has full rank, the Odd Zigzag inequalities are facet-inducing for $\operatorname{WGRP}\left(G_{\mathcal{C}}\right)$. Again, by applying the lifting theorem in Corberán, Plana \& Sanchis (2005), they are also facet-inducing for $\operatorname{WGRP}(G)$.

\section{Zigzag inequalities and other polyhedra.}

In this section we study the application of the Zigzag inequalities to other known arc routing polyhedra that are closely related to the WGRP. 


\begin{tabular}{|c|c|c|c|c|c|c|c|c|c|c|c|c|c|c|c|}
\hline & & & $\begin{array}{l}\text {-sel } \\
\text { apol }\end{array}$ & $\begin{array}{l}\text { cted } \\
\text { ents }\end{array}$ & & & $\begin{array}{c}\text { Other } \\
\text { non-selected }\end{array}$ & & & lect & ed c & $\mathrm{mp}$ & pner & & \\
\hline & & & in $J$ & & & & components & $(1,3)$ & $(3,1)$ & $(2,4)$ & $(4,2)$ & $(1,2)$ & $(2,3)$ & $(3,4)$ & $(4,1)$ \\
\hline 1 & -1 & 0 & 0 & & 0 & & & & & & & & & & \\
\hline 0 & 1 & 0 & 0 & $\ldots$ & 0 & 0 & & & & & & & & & \\
\hline 0 & 0 & 1 & -1 & $\ldots$ & 0 & 0 & & & & & & & & & \\
\hline 0 & 0 & 0 & 1 & $\ldots$ & 0 & 0 & 0 & & & & & & & & \\
\hline 0 & 0 & 0 & 0 & $\ldots$ & 1 & 1 & & & & & & & & & \\
\hline 0 & 0 & 0 & 0 & $\ldots$ & 0 & 1 & & & & & & & & & \\
\hline & & & 0 & & & & I & & & & & & & & \\
\hline & & & & & & & & 0 & 1 & 0 & 1 & 1 & 2 & 1 & 0 \\
\hline & & & & & & & & 1 & 1 & 1 & 0 & 1 & 0 & 0 & 1 \\
\hline & & & 0 & & & & 0 & 1 & 1 & 0 & 1 & 0 & 1 & 1 & 0 \\
\hline & & & & & & & & 1 & 0 & 1 & 1 & 0 & 0 & 1 & 1 \\
\hline & & & & & & & & 0 & 1 & 1 & 1 & 1 & 1 & 0 & 0 \\
\hline
\end{tabular}

Figure 4: Matrix appearing in the proof of theorem 4

\subsection{Windy Postman Problem}

When all the edges are required, the WGRP reduces to the WPP. In this case $\alpha_{i j}$ is the total number of edges in $\left(M^{i}, M^{j}\right)$. Note that the Even Zigzag inequalities do not apply to this problem, while the Odd Zigzag inequalities are valid and facet-inducing for $\operatorname{WPP}(G)$ under the conditions presented above. In the special case when $\mathcal{F}=\emptyset$, the Odd Zigzag inequalities are equivalent to the 3-wheel inequalities proposed by Win (1987) and hence they induce the same facets. To illustrate this, consider the Odd Zigzag configuration shown in figure 5a, where all the edges are required and the numbers represent the coefficients of the corresponding variables in the Zigzag inequality $F(x) \geq 6$. Due to the symmetry equation associated with node $M^{1}$, the sum $x_{21}+x_{41}+x_{31}$ in the inequality can be replaced by $x_{12}+x_{14}+x_{13}$ to obtain an equivalent inequality in which variables $x_{12}, x_{14}$ and $x_{13}$ now have coefficients 1,2 and 2, respectively, while variables $x_{21}, x_{41}$ and $x_{31}$ have coefficients 1,0 and 0 . We then proceed in a similar way with node $M^{2}$ and we obtain an equivalent inequality whose coefficients are shown in figure 5b. Dividing this inequality by 2 and representing only those arcs corresponding to variables with a non-zero coefficient, the 3 -wheel inequality $F^{\prime}(x) \geq 3$ by Win (1987) and its associated graph (shown in figure 5c) are obtained. However, note that when $\mathcal{F} \neq \emptyset$, Odd Zigzag inequalities presented here are a new class of facet-inducing inequalities for the WPP. In fact, it can be seen that the extreme point shown in Win's Thesis (Figure 4.8b, page 74), which satisfies all the 3 -wheel inequalities, violates the Odd Zigzag inequality defined by node sets $M^{1}=\{6\}$, $M^{2}=\{3,4,5\}, M^{3}=\{2\}$ and $M^{4}=\{1\}$ and by edge set $\mathcal{F}=\left\{e_{46}\right\}$.

\subsection{Arc Routing Problems on undirected graphs}

Given an instance $G=\left(V, E, c_{i j}, c_{j i}\right)$ of a Windy Arc Routing Problem, if $c_{i j}=c_{j i}$ is satisfied $\forall e=\{i, j\} \in E$, the instance can also be considered as an instance of the corresponding Undirected ARP. Obviously, the natural formulation for this instance uses only one variable per 


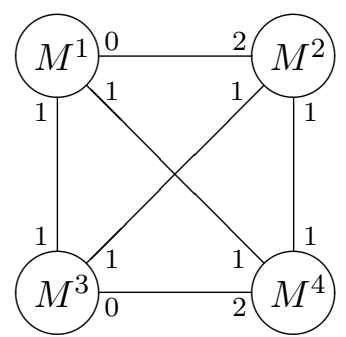

(a)

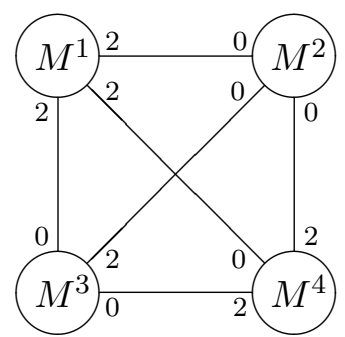

(b)

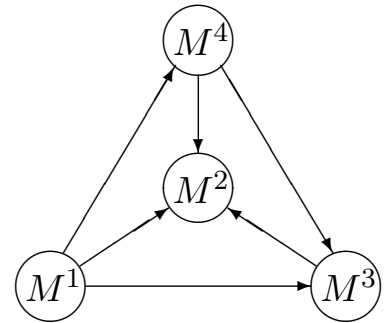

(c)

Figure 5: In the WPP the Odd Zigzag inequalities with $\mathcal{F}=\emptyset$ reduce to 3 -wheel inequalities

edge and the Zigzag inequalities do not apply. If a formulation with two variables per edge is used instead (handling an undirected instance as a windy instance), Zigzag inequalities are facet-inducing for the associated polyhedron.

\subsection{Arc Routing Problems on directed graphs}

An instance of an Arc Routing Problem defined on a directed graph can be transformed into an instance of the corresponding Windy ARP in which the cost of traversing the edges in the forbidden directions are set to infinity. The variables associated with the forbidden directions can then be removed from the Windy formulation and the natural formulation for the Directed ARP, with just one variable associated with each arc, is obtained. Hence, the Zigzag inequalities are valid for Directed ARPs. Nevertheless, it is not difficult to see that Odd Zigzag inequalities are dominated by flow inequalities and therefore they are not facet inducing for the directed ARP polyhedra.

Consider now the Even Zigzag inequalities. The fractional solution described in figure $2 \mathrm{~b}$ would be obtained for a DRPP instance similar to that in figure 2a with the appropriate arc directions. Therefore, the Even Zigzag inequalities do apply for the DRPP/DGRP:

Theorem 5 The Even Zigzag inequalities $F(x) \geq \alpha_{13}+\alpha_{24}+2$ are facet-inducing for DGRP $(G)$ if the following conditions are satisfied:

(a) there are at least four arcs forming the zigzag $M^{1}-M^{2}-M^{3}-M^{4}-M^{1}$,

(b) half of the $\alpha_{13}$ required arcs in $\left(M^{1}, M^{3}\right)$ are directed from $M^{1}$ to $M^{3}$ and the other half from $M^{3}$ to $M^{1}$. A similar condition is needed for the $\alpha_{24}$ required arcs in $\left(M^{2}, M^{4}\right)$.

Proof: The proof is similar to that of the WGRP and the details are omitted here. Just notice that the dimension of $\operatorname{DGRP}(G)$ is $|A|-|V|+1$ (see Corberán, Romero \& Sanchis, 2003) and the required arcs induce a symmetric subgraph.

\subsection{Arc Routing Problems on mixed graphs}

As on directed graphs, an instance of an Arc Routing Problem defined on a mixed graph can be transformed into an instance of the corresponding Windy ARP. Then, removing the variables associated with the forbidden directions, a formulation for the Mixed ARP, with just one variable associated with each arc and two variables associated with each edge, is obtained. This formulation, which we here call F2, is used, for example, by Christofides et al. (1984) and Ralphs (1993) for the MCPP. Nobert \& Picard (1996) and Corberán et al. (2003, 2005) propose 
a formulation for the MCPP and for the MRPP/MGRP, respectively, that uses only one variable for each edge which expresses the number of times a given edge is traversed (in any direction). We call this formulation F1. A theoretical and computational comparison of formulations F1 and F2 for Arc Routing Problems on mixed graphs can be found in Corberán, Mota \& Sanchis (2005).

Let us first consider formulation F2, which is more closely related to the windy formulation. It is easy to find two MGRP instances with fractional solutions similar to those shown in figures 1 and 2 for the WGRP. Hence, Zigzag inequalities apply to the MGRP. Given an MGRP instance $G=(V, E, A)$, where $V$ is the set of vertices, $E$ is the set of edges and $A$ is the set of arcs, the polyhedron associated with the set of solution vectors corresponding to formulation $\mathrm{F} 2$ is represented here as $\operatorname{MGRP}_{F 2}(G)$. It can be shown that its dimension is $2|E|+|A|-|V|+1$.

Obviously, Zigzag inequalities for the WGRP are valid for $\operatorname{MGRP}_{F 2}(G)$. Furthermore, as the following theorem states, they also have the facet inducing property if the required arcs have the appropriate direction.

Theorem 6 Zigzag inequalities are facet-inducing for $M G R P_{F 2}(G)$ if there are at least 4 links defining a zigzag $M^{1}-M^{2}-M^{3}-M^{4}-M^{1}$ and,

(a) for the even case: the required edges in $G_{\mathcal{C}}$ can be oriented in such a way that the required links induce a symmetric graph.

(b) for the odd case: there are two required edges $e_{13} \in\left(M^{1}, M^{3}\right)$ and $e_{24} \in\left(M^{2}, M^{4}\right)$, and the remaining required edges in $G_{\mathcal{C}}$ can be oriented in such a way that, together with the required arcs in $G_{\mathcal{C}}$, they induce a symmetric graph in which all the links in $(\mathcal{H} \cup \mathcal{D})_{R} \backslash \mathcal{F}$ are oriented in the direction of the zigzag and all the links in $\mathcal{F}$ are oriented in the opposite direction.

Proof: The proof is similar to that of theorems 2 and 4 and the details are omitted here.

Let us now consider formulation F1. It uses only one variable per edge, i.e. associated with each MGRP tour there is an incidence vector $y=\left(y_{e}: e \in E \cup A\right) \in \mathbb{Z}^{|E|+|A|}$, where $y_{e}$ denotes the number of times a link $e \in E \cup A$ appears in the tour. It is based on the characterization of an Eulerian mixed graph given by Ford \& Fulkerson (1962): $G$ is Eulerian if, and only if, $G$ is connected, even and balanced. A graph $G$ is called balanced if, for every $S \subset V$, the difference between the number of arcs leaving $S$ and the number of arcs entering $S$ is less than or equal to the number of edges in $\delta(S)$ (balanced-set condition for set $S$ ). As in Corberán et al. (2003, 2005 ), we assume that the transformation of the original MGRP instance mentioned in section 2 not only obtains $V=V_{R}$, but also $E_{N R}=\emptyset$ (basically replacing each non-required edge by two non-required arcs). Therefore, formulation F1 considers the MGRP to be defined on a strongly connected mixed graph $G=(V, E, A):=\left(V_{R}, E_{R}, A_{R} \cup A_{N R}\right)$. It is easy to see that the set of tours for the MGRP is then the set of vectors $y \in \mathbb{Z}^{|E|+|A|}$ satisfying:

$$
\begin{aligned}
y_{i j} \geq 1, & \forall(i, j) \in A_{R}, \forall\{i, j\} \in E_{R} \\
y\left(A^{+}(S)\right)-y\left(A^{-}(S)\right) \leq y(E(S)), & \forall S \subset V \\
y\left(A^{+}(S)\right) \geq 1, & \forall S=\cup_{k \in Q} V_{k}, \quad Q \subset\{1, \ldots, p\} \\
y(\delta(\{i\})) \equiv 0 \bmod 2, & \forall i \in V \\
y_{i j} \geq 0, & \forall(i, j) \in A_{N R} \\
y_{i j} \text { integer, } & \forall(i, j) \in A_{R} \cup A_{N R}, \forall\{i, j\} \in E_{R}
\end{aligned}
$$

where (11) implies that all the required links are in the solution, (12), (13) and (14) assure that the resulting graph will be balanced, connected and even, respectively. $A^{+}(S)\left(A^{-}(S)\right)$ 
represents the set of arcs leaving (entering) a vertex subset $S$, while $E(S)$ denotes the set of edges incident with $S$. Let $\left(S_{1}, S_{2}\right)$ denote the set of links (arcs and edges) with an endpoint in $S_{1}$ and another in $S_{2}$, let $A\left(S_{1}, S_{2}\right)$ be the set of arcs from $S_{1}$ to $S_{2}$ and $E\left(S_{1}, S_{2}\right)$ the set of edges between $S_{1}$ and $S_{2}$. A vertex is called $R$-even ( $R$-odd) if it is incident with an even (odd) number of required links. Given a set $H$ of links, let $y(H)$ be the sum of the variables associated with all the links in $H$.

The polyhedron associated with the set of solution vectors corresponding to formulation F1 is here called $\operatorname{MGRP}_{F 1}(G)$ and its dimension is $|E \cup A|-q+1$, where $q$ is the number of connected components of the graph $(V, E)$ (see Corberán, Romero \& Sanchis, 2003). The Zigzag configuration for $\operatorname{MGRP}_{F 1}(G)$ consists of a partition of the set of vertices $V$ into 4 parts, $M^{1}$, $M^{2}, M^{3}$ and $M^{4}$ satisfying:

Even case: Each $M^{1}, M^{2}, M^{3}$ and $M^{4}$ contains an even number of $R$-odd vertices and $\alpha_{12}=$ $\alpha_{34}=\alpha_{14}=\alpha_{23}=0$.

Odd case: Each $M^{1}, M^{2}, M^{3}$ and $M^{4}$ contains an odd number of $R$-odd vertices and the graph induced by the required links in $G_{C}$, plus two extra arcs $a_{12}$ and $a_{34}$ from $M^{1}$ to $M^{2}$ and from $M^{3}$ to $M^{4}$ respectively, is not a balanced graph.

Here, $\alpha_{i j}$ represents the number of required links in $\left(M_{i}, M_{j}\right)$. The configuration graph, $G_{\mathcal{C}}$, associated with the Zigzag inequalities is defined by the partition of $V$ above and by the following coefficients: $c\left(A\left(M^{1}, M^{2}\right)\right)=c\left(A\left(M^{3}, M^{4}\right)\right)=0, \quad c\left(A\left(M^{2}, M^{1}\right)\right)=c\left(E\left(M^{1}, M^{2}\right)\right)=$ $c\left(A\left(M^{4}, M^{3}\right)\right)=c\left(E\left(M^{3}, M^{4}\right)\right)=2, \quad c\left(M^{i}, M^{j}\right)=1$, otherwise. Figure 6 shows an odd configuration. The Zigzag inequality for $\operatorname{MGRP}_{F 1}(G)$ is:

$$
\begin{aligned}
& y\left(M^{1} \cup M^{2}, M^{3} \cup M^{4}\right)+2 y\left(\left(M^{1}, M^{2}\right) \backslash A\left(M^{1}, M^{2}\right)\right)+2 y\left(\left(M^{3}, M^{4}\right) \backslash A\left(M^{3}, M^{4}\right)\right) \geq \\
& \geq \alpha_{13}+\alpha_{24}+\alpha_{23}+\alpha_{14}+2\left|\left(M^{1}, M^{2}\right)_{R} \backslash A\left(M^{1}, M^{2}\right)\right|+2\left|\left(M^{3}, M^{4}\right)_{R} \backslash A\left(M^{3}, M^{4}\right)\right|+2
\end{aligned}
$$

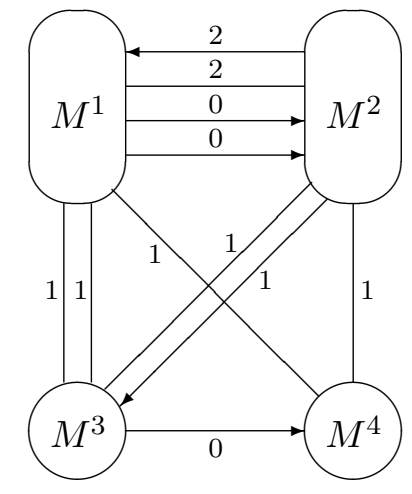

Figure 6: An Odd Zigzag configuration for the MGRP with formulation F1.

Theorem 7 Zigzag inequalities (17) are valid for $M G R P_{F 1}(G)$. Moreover they are facetinducing for $M G R P_{F 1}(G)$ if there are at least two arcs $a_{12} \in A\left(M^{1}, M^{2}\right)$ and $a_{34} \in A\left(M^{3}, M^{4}\right)$ and two other links defining a zigzag $M^{1}-M^{2}-M^{3}-M^{4}-M^{1}$ and:

(a) for the even case: the required links in $G_{\mathcal{C}}$ induce a balanced graph.

(b) for the odd case: there are two required edges $e_{13} \in\left(M^{1}, M^{3}\right), e_{24} \in\left(M^{2}, M^{4}\right)$ and the remaining required links in $G_{\mathcal{C}}$ induce a balanced graph. 
Proof: The proof that Zigzag inequalities are valid for $\operatorname{MGRP}_{F 1}(G)$ and facet-inducing for $\operatorname{MGRP}_{F 1}\left(G_{\mathcal{C}}\right)$ is similar to those in theorems 1 to 4 and the details are omitted here. Moreover, the lifting theorem in Corberán et al. (2005) can be applied to show that Even Zigzag inequalities are facet-inducing for $\operatorname{MGRP}_{F 1}(G)$. However, the same theorem cannot be applied for the odd case, since its first condition is not satisfied because of the need for the two required edges $e_{13} \in\left(M^{1}, M^{3}\right)$ and $e_{24} \in\left(M^{2}, M^{4}\right)$ in condition (b) above. Nevertheless, a slightly different lifting theorem can be specifically developed for the Odd Zigzag inequalities. Its proof is technical and is omitted here.

As in Corberán et al. (2003, 2005), formulation F1 can be expressed in terms of semitours. A semitour for the MGRP is the family of links $z$ obtained from any tour for the MGRP $y$ by deleting one copy of every required link, i.e. $y=z+y^{R}$, where $y^{R}$ is the incidence vector of the required links. Given that Zigzag inequality (17) can be written as $F\left(z+y^{R}\right) \geq F\left(y^{R}\right)+2$, the Zigzag inequality with respect to semitours is simply $F(z) \geq 2$.

Finally, we would like to point out that although Zigzag inequalities are facet-inducing for $\operatorname{MGRP}_{F 2}(G)$ and $\operatorname{MGRP}_{F 1}(G)$, there is not a one-to-one correspondence between these facets. Consider, for example, the MGRP instance $G$ shown in figure $7 \mathrm{a}$, in which all the links are required and numbers near the links represent their costs. While the polyhedron $\operatorname{MGRP}_{F 2}(G)$ has a facet induced by a Zigzag inequality, this is not the case for $\operatorname{MGRP}_{F 1}(G)$. Using the

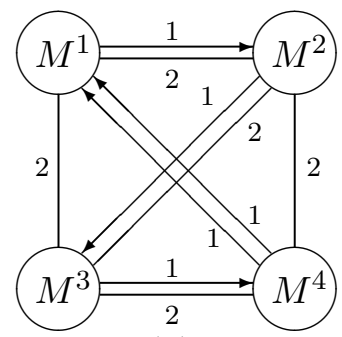

(a)

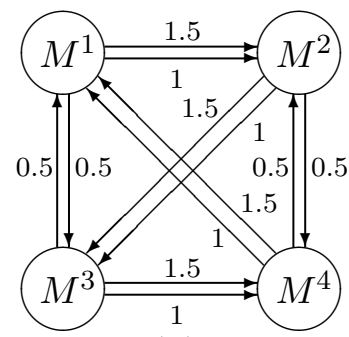

(b)

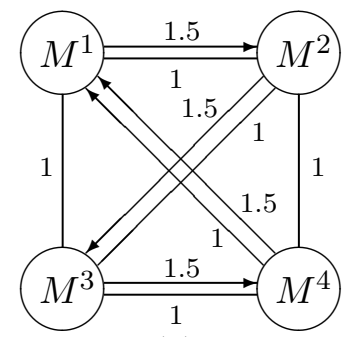

(c)

Figure 7: WGRP instance $G$ and fractional vectors

cutting-plane algorithm based on formulation F2 for the MGRP described in Corberán, Mota \& Sanchis (2005), the fractional solution shown in figure $7 \mathrm{~b}$ is obtained (numbers near the links represent their corresponding variable values). This solution does not satisfy the Zigzag inequality $F(x) \geq 8$. Note that this inequality is valid and facet-inducing for $\operatorname{MGRP}_{F 2}(G)$. On the other hand, it is easy to see that the corresponding Zigzag inequality with respect to formulation $\mathrm{F} 1, F(y) \geq 12$, is not valid for $\operatorname{MGRP}_{F 1}(G)$, but such an inequality is not needed here. Note that the corresponding fractional vector in terms of formulation F1 shown in figure $7 \mathrm{c}$ is not a solution of the cutting-plane algorithm since it is a convex linear combination of two MGRP tours.

\section{Computational experiments}

In order to know if the Zigzag inequalities are useful from a computational point of view, its associated separation problem has to be studied. At this moment we do not know if the problem of separating these inequalities can be solved in polynomial time or not. Answering this question and developing efficient exact or approximate separation algorithms do not seem easy tasks, and we want to deal with them in the near future. 
Here we have just tried to visually identify violated Zigzag inequalities on randomly generated WPP instances of small size. The reason for choosing the WPP is that, except for Win's oddwheel inequalities (and 3-wheel inequalities are a particular case of the Zigzag ones), all the other known facet inducing inequalities can be separated in polynomial time.

A cutting-plane procedure with separation algorithms for the Odd-cut inequalities is applied to each WPP instance. If the obtained solution is integral, it is an optimal tour. Otherwise, the fractional solution is depicted and we visually look for a violated Zigzag inequality. If none is found, the procedure stops. Otherwise, the violated inequality is added to the current LP and the procedure continues as usual.

The WPP instances are generated as follows. First, $|V| \in(10,19)$ vertices are randomly selected in a $1000 \times 1000$ square and the distances between all of them are computed as $c_{i j}=$ $\left\lfloor d_{i j}+0.5\right\rfloor$, where $d_{i j}$ are the Euclidean distances. Then, the $d \in(3,5)$ shortest edges incident with every vertex $i$ are added and those edges $\{i, j\}$ for which $c_{i j} \geq 0.98\left(c_{i k}+c_{k j}\right)$ for some $\{i, k\}$ and $\{k, j\}$ are removed. If the resulting graph is not connected, edges in the $d$ shortest trees spanning the connected components are also added. A total of 400 undirected connected graphs are built in this way to obtain two different sets of WPP instances. The difference between both test sets is the way in which edge costs are selected. The 200 instances in the first set have costs which are directly related to the Euclidean distances. The new costs $c_{i j}^{\prime}$ are computed from the original costs $c_{i j}$ by adding an integer number randomly selected in $(-a, a)$ (see Win, 1987), where parameter $a$ is a random percentage (between $15 \%$ and 30\%) of the average edge cost. The costs of the 200 instances in the second set are randomly generated in $(1,4)$.

\begin{tabular}{|lrccr|}
\hline instance & \multicolumn{1}{c}{ LB } & Zigzag1 & Zigzag2 & \multicolumn{1}{c|}{ LB $_{z z}$} \\
\hline wppe1 & 7177.5 & 1 & - & $7186^{*}$ \\
wppe2 & 10647.5 & - & 1 & $10651^{*}$ \\
wppe3 & 11618.0 & - & 1 & $11629^{*}$ \\
wppe4 & 7167.5 & 1 & - & $7196^{*}$ \\
\hline wppne1 & 46.5 & 1 & - & $47^{*}$ \\
wppne2 & 89.0 & - & 2 & 89.5 \\
wppne3 & 110.5 & - & - & \\
wppne4 & 77.5 & 1 & - & $78^{*}$ \\
wppne5 & 76.0 & - & 1 & $76^{*}$ \\
wppne6 & 85.5 & - & 1 & $86^{*}$ \\
\hline
\end{tabular}

Table 1: Computational results

The cutting-plane procedure was able to solve 196 out of 200 instances in the first set and 194 in the second set. Table 1 shows the computational results obtained for the 10 unsolved instances, where wppe 1 to wppe 4 are the instances with costs based on Euclidean distances and wppne 1 to wppne 6 are the instances with random costs. Column $L B$ gives the value of the lower bound obtained with the cutting-plane procedure, while $L B_{z z}$ shows the bound obtained after the addition of the violated Zigzag inequalities. A '*' means that not only the optimal value but also an optimal WPP solution was obtained. The number of Odd Zigzag inequalities found for each instance is shown in columns Zigzag1 (those equivalent to 3-wheel inequalities) and Zigzag2. Note that 8 instances were solved to optimality after the addition of a Zigzag inequality. For the instance wppne2, the addition of 2 Zigzag inequalities was not enough to obtain an optimal solution. Finally, we did not find any violated Zigzag inequality for the instance wppne3. The performance of the cutting-plane procedure was very good on these small size instances. We 
think that it will perform worse on larger instances, thus making the Zigzag inequalities more useful.
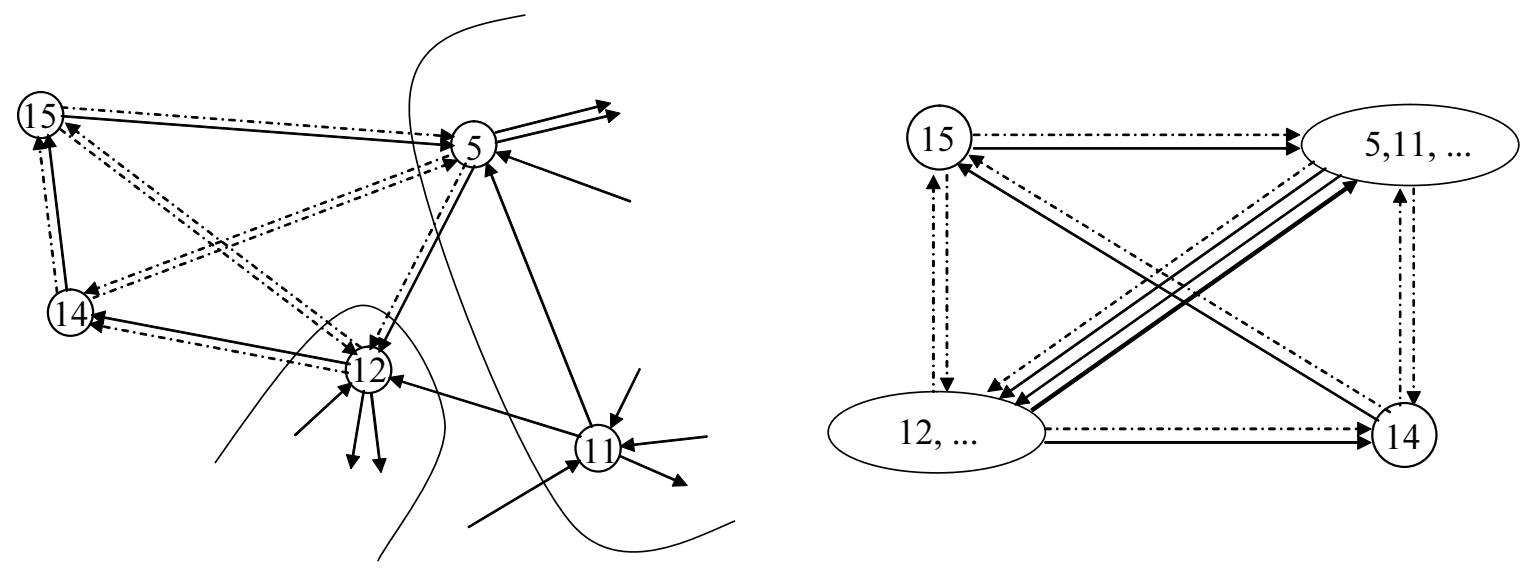

Figure 8: WPP fractional solution and Odd Zigzag configuration

Figure 8 shows part of the fractional solution obtained by the cutting-plane algorithm when applied to the instance wppne5, whose data are given in Table 2. As in previous figures, arcs drawn in solid lines correspond to variables with value 1 , arcs in dotted lines represent variables with value 0.5 , while variables with value 1.5 are drawn with a solid and a dotted arc simultaneously. This fractional solution violates the Odd Zigzag inequality

$$
\begin{aligned}
& x_{15,12}+x_{12,15}+x_{5,14}+x_{14,5}+x_{14,15}+x_{15,14}+x_{5,12}+x_{12,5}+ \\
+ & x_{11,12}+x_{12,11}+x_{2,11}+3 x_{11,2}+2 x_{5,15}+2 x_{14,12} \geq 8
\end{aligned}
$$

whose configuration is also shown in Figure 8, where the only edge in $\mathcal{F}, e=\{2,11\}$, is represented in bold. After adding this inequality, the cutting-plane algorithm was capable of finding the optimal solution.

\begin{tabular}{|ccc|}
\hline vertex & x-coord & y-coord \\
\hline 1 & 683 & 647 \\
2 & 290 & 439 \\
3 & 599 & 828 \\
4 & 946 & 88 \\
5 & 543 & 820 \\
6 & 926 & 313 \\
7 & 108 & 325 \\
8 & 187 & 357 \\
9 & 845 & 757 \\
10 & 764 & 584 \\
11 & 511 & 621 \\
12 & 330 & 759 \\
13 & 992 & 156 \\
14 & 161 & 860 \\
15 & 143 & 925 \\
\hline
\end{tabular}

\begin{tabular}{|ccc|ccc|}
\hline$\{i, j\}$ & $c_{i j}$ & $c_{j i}$ & $\{i, j\}$ & $c_{i j}$ & $c_{j i}$ \\
\hline$\{1,3\}$ & 1 & 2 & $\{1,4\}$ & 2 & 4 \\
$\{1,5\}$ & 3 & 4 & $\{1,9\}$ & 2 & 4 \\
$\{1,10\}$ & 3 & 1 & $\{1,11\}$ & 3 & 3 \\
$\{2,8\}$ & 2 & 1 & $\{2,11\}$ & 3 & 4 \\
$\{2,12\}$ & 4 & 3 & $\{3,5\}$ & 3 & 1 \\
$\{3,9\}$ & 1 & 1 & $\{3,10\}$ & 1 & 2 \\
$\{3,11\}$ & 4 & 2 & $\{4,6\}$ & 2 & 2 \\
$\{4,10\}$ & 2 & 4 & $\{4,13\}$ & 1 & 1 \\
$\{5,11\}$ & 4 & 1 & $\{5,12\}$ & 2 & 2 \\
$\{5,14\}$ & 1 & 3 & $\{5,15\}$ & 1 & 1 \\
$\{6,10\}$ & 3 & 3 & $\{6,13\}$ & 4 & 4 \\
$\{7,8\}$ & 3 & 1 & $\{7,12\}$ & 1 & 2 \\
$\{8,12\}$ & 4 & 2 & $\{9,10\}$ & 4 & 3 \\
$\{10,11\}$ & 4 & 1 & $\{11,12\}$ & 2 & 2 \\
$\{12,14\}$ & 1 & 4 & $\{12,15\}$ & 1 & 3 \\
$\{14,15\}$ & 2 & 2 & & & \\
\hline
\end{tabular}

Table 2: WPP instance data 


\section{Conclusions}

In this paper a new class of facet-inducing inequalities for several well known Arc Routing Problems is introduced, the Zigzag inequalities. Two different versions, the odd case and the even case, are presented. While Even Zigzag inequalities are configuration inequalities, Odd Zigzag ones are, as far as we know, the first described class of weak configuration inequalities that induce facets for an Arc Routing Polyhedron.

In summary, Even Zigzag inequalities are facet-inducing for the polyhedra associated with the Directed Rural Postman Problem, Directed General Routing Problem, Mixed Rural Postman Problem (F1 and F2), Mixed General Routing Problem (F1 and F2), Windy Rural Postman Problem and Windy General Routing Problem. Odd Zigzag inequalities are proved to be facet-inducing for the polyhedra associated with the Mixed Chinese Postman Problem (with formulations F1 and F2), Mixed Rural Postman Problem (F1 and F2), Mixed General Routing Problem (F1 and F2), Windy Postman Problem (where they generalize Win's 3-wheel inequalities), Windy Rural Postman Problem and Windy General Routing Problem.

Acknowledgments: The authors wish to thank the Ministerio de Ciencia y Tecnología of Spain (project TIC2003-05982-C05-01) and the Generalitat Valenciana (Ref: GRUPOS03/189) their support.

\section{References}

[1] E. Benavent, A. Carrotta, A. Corberán, J.M. Sanchis \& D. Vigo (2003): "Lower Bounds and Heuristics for the Windy Rural Postman Problem". Technical Report TR03-2003. Department of Statistics and OR, University of Valencia (Spain). Submitted to EJOR.

[2] E. Benavent, A. Corberán, E. Piñana, I. Plana \& J.M. Sanchis (2005): "New Heuristics for the Windy Rural Postman Problem". To appear in Computers $\&$ Operations Research.

[3] S. Chopra \& G. Rinaldi (1996): "The Graphical Asymmetric Traveling Salesman Polyhedron: Symmetric Inequalities". SIAM J. Discrete Math. 9, 4, 602-624.

[4] N. Christofides, E. Benavent, V. Campos, A. Corberán \& E. Mota (1984): "An Optimal Method for the Mixed Postman Problem". In P. Thoft-Christensen (Ed.) System Modelling and Optimization. Lecture Notes in Control and Information Sciences, 59. Berlin: SpringerVerlag.

[5] N. Christofides, V. Campos, A. Corberán \& E. Mota (1981): "An Algorithm for the Rural Postman Problem". Report IC.OR. 81.5. Imperial College, London.

[6] A. Corberán, G. Mejía \& J.M. Sanchis (2005): "New Results on the Mixed General Routing Problem". To appear in Operations Research.

[7] A. Corberán, E. Mota \& J.M. Sanchis (2005): "A Comparison of Two Different Formulations for Arc Routing Problems on Mixed Graphs". To appear in Computers 83 Operations Research. 
[8] A. Corberán, I. Plana \& J.M. Sanchis (2005): "On the Windy General Routing Polyhedron". In preparation.

[9] A. Corberán, A. Romero \& J.M. Sanchis (2003): "The Mixed General Routing Problem Polyhedron". Mathematical Programming, 96, 103-137.

[10] G. Cornuèjols, J. Fonlupt \& D. Naddef (1985): "The traveling salesman problem on a graph and some related integer polyhedra". Mathematical Programming 33, 1-27.

[11] H.A. Eiselt, M. Gendreau \& G. Laporte (1995): "Arc-Routing Problems, Part 2: the Rural Postman Problem". Operations Research, 43, 399-414.

[12] L.R. Ford \& D.R. Fulkerson (1962): Flows in Networks. Princeton University Press, Princeton, NJ.

[13] M. Grötschel \& Z. Win (1988): "On the Windy Postman Polyhedron". Report No. 75, Schwerpunktprogram der Deutschen Forschungsgemeinschaft, Universität Augsburg, Germany.

[14] M. Grötschel \& Z. Win (1992): "A Cutting Plane Algorithm for the Windy Postman Problem". Mathematical Programming 55, 339-358.

[15] M. Guan (1984): "On the Windy Postman Problem". Discrete Applied Mathematics, 9, 41-46.

[16] A. Letchford (1997): "New inequalities for the General Routing Problem". European Journal of Operational Research, 96, 317-322.

[17] E. Minieka (1979): "The Chinese Postman Problem for Mixed Networks". Management Science 25, 643-648.

[18] D. Naddef \& G. Rinaldi (1991): "The Symmetric Traveling Salesman Polytope and its Graphical Relaxation: Composition of Valid Inequalities". Mathematical Programming 51, 359-400.

[19] Y. Nobert \& J.C. Picard (1996): "An Optimal Algorithm for the Mixed Chinese Postman Problem". Networks 27, 95-108.

[20] T.K. Ralphs (1993) : "On the Mixed Chinese Postman Problem". Operations Research Letters 14, 123-127.

[21] Z. Win (1987): "Contributions to Routing Problems". PhD Dissertation, University of Augsburg, Germany. 